$u=\theta(f)$ vérifie la même condition et, par suite, elle est absolument $\operatorname{contin} \theta^{1}$ ). La fonction

$$
f=\theta^{-1}(u)=\varphi(u)
$$

est ainsi une f. a. c. d'une f. a. c.

Remarque. On peut aieément déduire de l'énoncé qu'on vient de prouver la proposition de Mlle Bary et de M. Menchoff. Soit, à cet effèt, $f(x)$ une fonction dans $(0,1)$ satisfaisant à la condition de ces auteurs. En désignant donc par $M$ l'ensemble de tous les points $x$ où $f(x)$ n'admet pas la dérivée finie et unique, on a:

(1)

$$
\operatorname{mes} M_{f}=0 \text {. }
$$

Soit $E$ un ensemble quelconque dans $(0,1)$ de mesure nulle. $f(x)$ admettant la dérivée en chaque point de $E-M$, l'ensemble $(E-M)$ s est, en vertu d'an thérereme de M. Lugin '), de mesure nulle. Donc, en verta de (1), $E\left(\left(E-M_{f}+M_{f}\right.\right.$ est aussi de mescre nulle, et la fonction $f(x)$ possède la propriété $(N)$.

Soit maintenant $A$ l'ensemble de valeurs $y$ telles que $N_{f}(y)$ y est infini. A chaque valuur $y \& A$ on peut donc faire correspondre un point $x_{y}$ qui soit un point d'accumulation d'une suite de points $x_{y}^{(n)}$ tels que $f\left(x_{y}^{(n)}\right)=y$. Désignons par $B$ l'ensemble de valeurs $y \varepsilon A$ pour lesquels $x_{y} \varepsilon M$. On a donc

$$
B C M_{f} \text {, donc: } \operatorname{mes} B=0 \text {. }
$$

D'autro part, si $x_{y}$ n'appartient pas à $M$, la dérivée $f^{\prime}\left(x_{v}\right)$ existant an ce point, elle s'y annule nécessairement et, par conséquent, l'ensemble $A-B$ de valeurs correspondant aux tels points eqt de mesure nulle $\left.{ }^{3}\right)$. Donc, d'uprès (2), on a: $\operatorname{mes} A=0$, c.-̀.-d. $f(x)$ satisfait encore à la condition $(T)$.

Les conditions $(N)$ et $(T)$ vérifiées par la fonction $f(x)$, elle est d'après lo théorème précédent une f. a. c. d'une f. a. c.

Remarque post seriptum. Après l'envoi par nous de la pré sente note à la Rédaction des „Fundamenta ${ }^{\prime}$, Mlle Bary a publié une nouvelle Note (C. R. t. 183. p. 469) où elle a signalé (sans démonstration) la même proposition que nous venons d'obtenir. $\mathrm{Or}$, la démonstration de Mlle Bary étant (d'après les renseignements que Mlle Bary a bien voulu nous faire parvenir) essentiellement différente de lä nôtre, nous croyons de pouvoir publier cette dernière, d'autant plus qu'elle semble etre aussi simple que naturelle.

Les raisonnements complets de Mlle Bary et de M. Menchoff paraîtront dans le recueil italien ${ }_{n} \mathrm{Annali}$ di Matematica".

1) Bau ach, 1. c. p. 229.

3) Lusin, L'intégrale et la série trigonométrique (en rasse) Moscou. 1915. p. 105. Cf. ausii: Saks. Sur un théorème de M. Lasin. Fund. Math. 192 vol. VI p. 111 .

2) 1. c.

\section{Sur les projections des ensembles complémentaires} aux ensembles $(A)$.

Par

\section{W. Sierpiński (Varsovie).}

Le but de cette Note est de démontrer la proposition suivante: Théorème: Il existe un ensemble de nombres réels $H$, complémentaire d'un ensemble (A), tel que tout ensemble linéaire qui est une projection d'un ensemble plan complémentaire d'un ensemble (A) est une image univoque et continue de l'ensemble $H$ (et inversement).

Après avoir démontré ce théorème, nous indiquerons sa généralisation concernant les ensembles projectifs de M. L usin.

Soit $U$ un ensemble analytique universel (plan), c'est-à-dire un ensemble $(A)$ de points du plan $X O Y$, tel qu'on a en le coupant avec les drojtes parallèles à l'axe $O Y$ tous les ensembles (A) linéaires possibles ${ }^{1}$ ). Soit $V=C U$ le complémentaire de $U$ (par rapport. au plan $X O Y$ ): ce sera évidemment un ensemble plan $C A$ universel.

Le plan $X O Y$, en tant qu'un ensemble $(A)$, est, comme on sait, une image univoque et continue $f(N)^{2}$ ) de l'ensemble $N$ de tous les nombres irrationnels. Désignons par $H$ l'ensemble de tous les nombres $x$ de $N$, pour lesquels $f(x)$ appartient à $V^{3}$ ).

1) N. Lusin: Fund. Wath. t. X, p. 79. Cf aussi: W. Sierpinski: Fund. Math. t. VII, p. 201.

2) $g$ étant une fonction définie dans l'ensemble $E, g(E)$ désigne l'ensemble de toutes les valeuris que prend $g$ dans $E$.

s) L'ensemble $H$ pourrait être dófini effectivement. 
Je dis que l'ensemble $H$ satisfait aux conditions de notre théorème.

Nous prouverons d'abord que l'ensemble $H$ est complémentaire d'un ensemble $(A)$, ou, ce qui revient au même, que l'ensemble $N-H$ est un ensemble $(A)$.

Désignons, pour tout nombre $z$ de $N$, par $\varphi(z)$ et $\psi(z)$ l'abscisse et l'ordonnée du point $f(z)$ (du plan $X O Y$ ) et considérons l'ensemble $M$ de tous les points $(\varphi(z), \psi(z), z)$ de l'espace, tels que $z \varepsilon N$. Les fonctions $\varphi(\dot{z})$ et $\psi(z)$ étant continues dans $N$, on roit sans peine que $M$ est un ensemble $(A)$ à trois dimensions (même un $G_{\delta}$ ). Or, désignons par $T$ l'ensemble de tous les points $(x, y, z)$ de l'espace, tels que $(x, y) \varepsilon U$. $U$ étant un ensemble $(A), T$ sera aussi un ensemble $(A)$. L'ensemble $M T$ sera donc un $(A)$ (comme produit de deux ensembles $(A)$ ), en même temps que sa projection sur l'axe $O Z$. Or, la projection de l'ensemble $M T$ sur l'axe $O Z$ coincide, comme on voit sans peine, avec l'ensemble $N-H$ (situé sur l'axe $O Z$ ). Ce dernier est done un ensemble $(A)$, c. q. f. d.

Lemme. Soit $X$ un ensemble de nombres irrationnels, $X_{1}$ - un sousensemble de $X$ fermé dans $X, f(x)$-une fonction réelle définie dans $X_{1}$. On peut toujours définir dans $X$ une fonction réelle $\varphi(x)$, continue dans $X$, telle que $\varphi(x)=f(x)$ dans $X_{1}$ et que $\varphi(X)=f\left(X_{1}\right)$.

Démonstration. L'ensemble $C \bar{X}_{1}$ (le complémentaire de la fermeture de l'ensemble $X_{1}$ ) est ouverh done il est une somme d'un nombre fini ou d'une infinité dénombrable d'intervalles ouverts (finis ou infinis) n'empiétant pas les uns sur les autres:

$$
c \bar{X}_{1}=\delta_{1}+\delta_{2}+\delta_{3}+\ldots
$$

Dans chacun des intervalles

$$
\delta_{n}\left(\alpha_{n}<x<\beta_{n}\right)
$$

choisissons un nombre rationnel $r_{n}$.

$\mathrm{Si}-\infty<\alpha_{n}$, on a évidemment $\alpha_{n} \varepsilon \bar{X}_{1} ;$ pareillement, si $\beta_{n}<+\infty$, on a $\beta_{n} \varepsilon \bar{X}_{1}$. L'ensemble $X_{1}$ étant fermé dans $X$, il en résulte tout de suite que si $\alpha_{n} \varepsilon X$, on a $\alpha_{n} \varepsilon X_{1}$, et si $\beta_{n} \varepsilon X$, on a $\beta_{n} \varepsilon X_{1}$.

Choisissons dans $X_{1}$ un nombre fixe $\xi$.

Posons $\varphi(x)=f(x)$ dans $X_{1}$.
Soit maintenant $x$ un nımbre de $X-X_{1}$. L'ensemble $X_{1}$ étant fermé dans $X$, on a, comme on voit sans peine:

$$
X-X_{1}=X . C \overline{X_{1}}:
$$

d'après (1) il existe donc un indice $n$, tel que $x \varepsilon \delta_{n}$, c'est-à-dire $\alpha_{n}<x<\beta_{n}$. Or, $x$, comme élément de l'ensemble $X$, est un nombre irrationnel, et par suite $x \neq r_{n}$ : d'après $\alpha_{n}<x<\beta_{n}$ nous avons donc soit $\alpha_{n}<x<\dot{r}_{n}$, soit $r_{n}<x<\beta_{n}$. Distinguons donc deux cas:

1) $\alpha_{n}<x<r_{n}$

Si $\alpha_{n} \otimes X_{1}$, posons $\varphi(x)=f\left(a_{n}\right)$.

Si $\alpha_{n}$ non $\varepsilon X_{1}, \alpha_{n} \neq-\infty$, on a $\alpha_{n} \varepsilon \bar{X}_{1}$ et il existe un nombre $a_{n}$ de $X_{1}$, tel que $\left|a_{n}-a_{n}\right|<r_{n}-a_{n}$. Posons dans ce cas $\varphi(x)=f\left(a_{n}\right)$.

Si $\alpha_{n}=-\infty$, posons $\varphi(x)=f(\xi)$.

2) $r_{n}<x<\beta_{n}$.

Si $\beta_{n} \varepsilon X_{1}$, posons $\varphi(x)=f\left(\beta_{n}\right)$.

Si $\beta_{n}$ non $\varepsilon X_{1}, \beta_{n} \neq+\infty$, on a $\beta_{n} \varepsilon \bar{X}_{1}$ et il existe un nombre $b_{n}$ de $X_{1}$, tel que $\left|b_{n}-\beta_{n}\right|<\beta_{n}-r_{n}$. Posons dans ce cas $\varphi(x)=f\left(b_{n}\right)$. Si $\beta_{\mathrm{n}}=+\infty$, posons $\varphi(x)=f(\xi)$.

La fonction $\varphi(x)$ est ainsi définie dans l'ensemble $X$ et on a évidemment $\varphi(X)=f\left(X_{1}\right)$. Il reste donc a démontrer que la fonction $\varphi(x)$ est enntinue dans $X$. Nous prouverons que la fonction $\varphi(x)$ est dans $X$ continue du côté gauche en tout point de $X$ : la démonstration qu'elle est continue dans $X$ du côté droit sera tout à fait analogue.

Soit $x_{0}$ un nombre de $X_{1}$. Nous avons donc $\varphi\left(x_{0}\right)=f\left(x_{0}\right)$.

Soit $\sigma$ un nombre positif donné. La fonction $f(x)$ étant continue dans $X_{1}$, il existe un nombre $\eta>0$, tel que

(2) $\quad\left|f(x)-f\left(x_{0}\right)\right|<\sigma$ pour $x \varepsilon X_{1}, \quad\left|x-x_{0}\right|<2 \eta$,

ce qui donne, la fonction $\varphi(x)$ étant égale à $f(x)$ dans $X_{1}$ :

(3) $\left|\varphi(x)-\varphi\left(x_{0}\right)\right|<\sigma$ pour $x \varepsilon X_{1}, \quad x_{0}-2 \eta<x<x_{0}$.

Distinguons maintenant deux cas.

1) Il existe un nombre $x_{1}$ de $X_{1}$, tel que

$$
x_{0}-\eta<x_{1}<x_{0}
$$

Soit $x$ un nombre de $X-X_{1}$, tel que

$$
x_{1}<x<x_{0} \text {. }
$$


D'après $x \varepsilon X-X_{1}$ on $\mathrm{a}$, comme nous savons, $x \varepsilon \delta_{n}$ (où $n$ est un nombre naturel), c'est-à-dire $\alpha_{n}<x<\beta_{n}$ et, l'intervalle $\left(\alpha_{n}, \beta_{n}\right)$ ne contenant à son intérieur aucun point de $X_{1}$, nous avons, d'après (5) ( $x_{0}$ et $x_{1}$ appartenant à $\left.X_{1}\right)$ :

$$
x_{1} \leqslant \alpha_{n}<\beta_{n} \leqslant x_{0} .
$$

Or, d'après la définition de la fonction $\varphi$ pour $\alpha_{n}<x<\beta_{u}$. on a soit $\varphi(x)=f\left(\alpha_{n}\right)$, soit $\varphi(x)=f\left(\beta_{n}\right)$, soit $\varphi(x)=f\left(\gamma_{n}\right)$, où $\left|\gamma_{n}-\alpha_{n}\right|<\beta_{n}-\alpha_{n}$, ou bien $\left|\gamma_{n}-\beta_{n}\right|<\beta_{n}-\alpha_{n}$. Donc, d'après (4) et (6), nous avuns dans tous les cas:

$$
\varphi(x)=f\left(x^{\prime}\right), \quad \text { ò̀ } \quad x^{\prime} \varepsilon X_{1} \text { et }\left|x^{\prime}-x_{0}\right|<2 \eta .
$$

ce quii donne, d'après (2) (et d'après $\varphi\left(x_{0}\right)=f\left(x_{0}\right)$ ):

$$
\left|\varphi(x)-\varphi\left(x_{0}\right)\right|<\sigma .
$$

Nous avons done démontré que l'inégalité (5) entraîne l'inégalité (7) pour $x \varepsilon X-X_{1}$. Or. d'après (3) et (4), l'inégalité (5) entrầne aussi l'inégalité (7) pour $x \varepsilon X_{1}$. Nous avons donc

$$
\left|\varphi(x)-\varphi\left(x_{0}\right)\right|<\sigma \text { pour } x \varepsilon X, \quad x_{1}<x<x_{0},
$$

ce qui prouve que la fonction $\varphi(x)$ est continue dans $X$ du côté gauche au point $x_{0}$.

2) Aucun nombre contenu entre $x_{0}-\eta$ et $x_{0}$ n'appartient pas à $X_{1}$. Dans ce cas, comme on voit sans peine, il existe un indice $n$, tel que $x_{0}=\beta_{n}$. D'après la définition de la fonction $\varphi$ on a donc

$$
\varphi(x)=f\left(\beta_{n}\right)=f\left(x_{0}\right)=\varphi\left(x_{0}\right) \text { pour } r_{n}<x<\beta_{n}=x_{0}
$$

ce qui prouve que la fonction $\varphi$ est continue dans $X$ du côté gauche au point $x_{0}$.

Soit maintenant $x_{0}$ un nombre de $X-X_{1}$. Il. existe done un indice $n$, tel que $\alpha_{n}<x_{0}<\beta_{n}$. Distinguons deux cas:

1) $\alpha_{n}<x_{0}<r_{n}$. D'après la définition de la fonction $\varphi$ on conclut tout de suite que dans ce cas:

$$
\varphi(x)=\varphi\left(x_{0}\right) \quad \text { pour } x \varepsilon X, \quad \alpha_{n}<x<r_{n} .
$$

2) $r_{n}<x_{0}<\beta_{n}$. D'après la définition de la fonction $\varphi$ nous trouvons dans ce cas:

$$
\varphi(x)=\varphi\left(x_{0}\right) \text { pour } x \varepsilon X, \quad r_{n}<x<\beta_{n} .
$$

La fonction $\varphi$ est done toujours continue dans $X$ du côté gauche au point $x_{0}$.

Notre lemme est ainsi démontré.

Soit maintenant $E$ un ensemble linéaire donné quelconque qui est une projection d'un ensemble plan, complémentaire à un ensemble ( $A$ ). Comme j'ai démontré ${ }^{1}$ ), l'ensemble $E$ est alors une image univoque et continue d'un ensemble linéaire $K$, complémentaire à un ensemble $(A)$. L'ensemble $V$ étant un ensemble (plan) $(' A$ universel, il existe un nombre $x_{1}$, tel qu'en coupant l'ensemble $V$ par la droite $x=x_{1}$ on obtient un ensemble $K_{1}$ superposable avec l'ensemble $K$. Or, l'ensemble $K_{1}$ est évidemment fermé dans $V$ : la fonction $f^{\prime}$ (pour laquelle $f(H)=V$ ) étant continue dans $H$, il en résulte que l'ensemble $H_{1}$ de tous les nombres $x$ de $H$, tels que $f(x) \varepsilon K_{1}$, est fermé dans $H$. D'après notre lemme il existe donc une fonction $\varphi(x)$, continue dans $H$, telle que $\varphi(x)=f(x)$ dans $B_{1}$ et que $\varphi(H)=f\left(H_{1}\right)$. Or, on a évidement $f\left(H_{1}\right)=K_{1}$ : done l'ensemble $K_{1}$, et parsuite aussi l'ensemble $K$ qui est superposable avec $K_{1}$, est une image univoque et continue de l'ensemble $H$. L'ensemble $E$ étant une image univoque et continue de l'ensemble $K$, il en resulte que $E$ est une image univoque et continue de l'ensemble $H$.

Or, soit $E$ un ensemble linéaire qui est une image univoque et continue de l'ensemble $\Psi$. L'ensemble $H$ étant un $C A$, il en résulte que $E$ est une projection orthogonale sur l'axe $O X$ d'un ensemble plan complémentaire à un ensemble $(A)^{1}$ ).

Nous avons ainsi démontré que les ensembles linéaires qui sont des projections des ensembles plans complémentaires aux ensembles $(A)$ coïncident avec les images univoques et continues de l'ensemble $H$. Notre théorème est ainsi démontré.

Nous indiquerons maintenant une extension de notre théorème aux ensembles projectifs de M. N. Lusin.

Considérons les ensembles projectifs (linéaires) de classe $n$. Nous appellerons $\dot{P}_{n}$ ceux des ensembles projectifs de classe $n$ qui sont de la forme $P C \ldots P E$, où $E$ est un ensemble mesurable $B$ (situé dans l'espace à $n+1$ dimensions), et par $C_{n}$ ceux qui sont de la

1) Fund. Math. t. VII, p. 239. 
forme $\left.C P C \ldots P E^{1}\right)$. Les ensembles $P_{1}$ coïncident donc avec les ensembles $(A)$ (linéaires), les ensembles $P_{2}$ - avec les ensembles qui sont projections des ensembles complémentaires aux ensembles $(A)$. On démontre dans la théorie des ensembles projectifs qu'il existe un ensemble $P_{n}$ (plan) universel ${ }^{2}$ ). En utilisant cet ensemble et en modifiant légèrement notre démonstration, on arrive au théorème suivant:

Il existe pour tout nombre naturel $n$ un ensemble de nombres réels $B_{n}$ qui est un $C_{n-1}$, tel que tout ensemble linéaire $P_{n}$ est une image univoque et continue de l'ensemble $H_{n}$ (et inversement).

Il est à remarquer qu'il n'existe. aucun ensemble linéaire $H_{\omega}$ qui jouirait de la propriété suivante: tout ensemble projectif (linéaire) est une image univoque et continue de l'ensemble $H_{\omega}$ et inversement. (En effet, un tel ensemble $H_{\omega}$ devrait être lui-même projectif, done un $P_{n}$. Or, on démontre dans la théorie des ensembles projectifs. que les images univoques et continues des ensembles $P_{n}$ sont encore des ensembles $P_{n}$ ). Or, on peut démontrer qu'il existe un ensemble linéaire $S$ (non projectif), tel que tout ensemble linéaire qui est une somme d'une infinité dénombrable d'ensembles projectifs est une image univoque et continue de l'ensemble $S$ et inversement.

1) Quant à la définition de ces ensembles, voir N̦. Lusin Fund. Math. t. X, p. 90. Les ensembles $P_{n}$ cö̈cident avec les ensembles de la classe $\mathscr{O} \mathcal{R}_{2 n+2}$ que j'ai introduit dans ma note des Fund. Math. t VII, p. 237.

2) Cela résulte tout de suite de l'existence d'un ensemble $(A)$ universol dans l'espace à $n>1$ dimensions (Cf. N. Lu sin, Fund. Math. t. X, p. 80, Remarque I. W. Sierpingki, Fund. Math. t. VIl, p. 241).

\section{Sur les produits des images continues des en- sembles $C(A)$. \\ Par

\author{
W. Sierpiński (Varsovie).
}

Le but de cette Note est de démontrer ce

Théorème: Si $E=E_{1} E_{2} E_{3} \ldots$, où $E_{n}(n=1,2,3, \ldots)$ sont des ensembles linéaires qui sont des images continues des ensembles linéaires $C A^{1}{ }^{1}$, l'ensemble $E$ est de même nature.

L'idée de notre démonstration sera basée sur celle, par laquelle M. N. Lusin a démontré que le produit d'une infinité dénombrable d'ensembles analytiques est un ensemble analytique ${ }^{2}$ ). Elle s'applique encore anx ensembles projectifs des classes supérieures.

Soit $n$ un nombre naturel donné. D'après l'hypothèse sur les ensembles $E_{n}$, il existe un ensemble $C A$ linéaire, soit $Q_{n}$, et une fonction $g_{n}(x)$, continue dans $Q_{n}$ telle que $\left.E_{n}=g_{n}\left(Q_{n}\right)^{s}\right)$. L'ensemble $X$ de tous les nombres réels est, comme on sait, une image univoque et continue de l'ensemble $N$ de tous les nombres irrationnels de l'intervalle $(0,1)$. Il existe donc une fonction $h(x)$, continue dans $N$, telle que $h(N)=X$. Désignons par $X_{n}$ l'ensemble de tous les nombres $x$ de $N$, tels que $h(x) \varepsilon Q_{n} ; Q_{n}$ étant un $C A$ et la fonotion $h(x)$ étant continue dans $N$, on voit sans peine que $X_{n}$ est un ' $C A$. Or, on a éridemment $h\left(X_{n}\right)=Q_{n}$, donc $E_{n}=g_{n}\left(h\left(X_{n}\right)\right)$, et la fonction $f_{n}(x)=g_{n}(h(x))$ est continue dans $X_{n}$.

Il existe donc, pour tout nombre naturel $n$, un ensemble $X_{n}$

1) On apelle ensembles $C A$ les ensembles complémentaires aux ensembles $(A)$

2) Fund. Math. t. X, p. 40 ot 41.

3) $g$ étant une fonction, définie dans l'ensemble $Q, g(Q)$ désigne l'ensemble de toutes les valeurs que prend $g$ dans $Q$ 